\title{
Snacking patterns of Irish adolescents (13-17 years of age)
}

\author{
M. Walsh, A. P. Nugent, A. P. Hearty and M. J. Gibney \\ UCD Institute of Food and Health, University College Dublin, Belfield, Dublin 4, Republic of Ireland
}

Energy intake from snacks in adolescents is reported to be between $20 \%$ and $33 \%{ }^{(1,2)}$. Using data from the National Teens Food Survey ${ }^{(3)}$ (NTFS), the snacking patterns of Irish teenagers was examined. Meal types were self-defined by the respondent and were aggregated into ten categories: (1) Breakfast; (2) lunch 'light meal'; (3) lunch 'main meal'; (4) evening meal 'light meal'; (5) evening meal 'main meal'; (6) morning snack; (7) afternoon snack; (8) evening snack; (9) non-alcoholic beverage; (10) alcoholic beverage. Morning, afternoon and evening snacks were combined to describe 'all snacks' and all meal types were combined to describe 'all meals'. All ten meal and snack types were collectively termed 'all eating occasions'. Food and nutrient intakes were assessed from all eating occasions, meals and snacks based on energy values $>0 \mathrm{~kJ}$.

\begin{tabular}{|c|c|c|c|c|c|c|}
\hline \multirow[b]{2}{*}{ Nutrients } & \multicolumn{2}{|c|}{ All eating occasions } & \multicolumn{2}{|c|}{ All meals } & \multicolumn{2}{|c|}{ All snacks } \\
\hline & Mean & SD & Mean & SD & Mean & SD \\
\hline No. of consumers & 441 & & 441 & & 436 & \\
\hline Total energy (MJ) & 8.33 & 2.44 & $6.14 *$ & 1.90 & 2.12 & 1.30 \\
\hline Protein (\% energy) & 14.8 & 2.57 & $16.9 *$ & 2.94 & 8.72 & 3.27 \\
\hline Total fat (\% energy) & 35.7 & 5.04 & $35.7^{\mathrm{NS}}$ & 5.47 & 36.2 & 7.80 \\
\hline Saturated fat (\% energy) & 14.4 & 2.69 & $13.8 *$ & 2.76 & 16.4 & 4.86 \\
\hline Carbohydrate (\% energy) & 52.3 & 5.44 & $50.3 *$ & 6.03 & 58.1 & 8.76 \\
\hline Added sugar (\% energy) & 13.3 & 5.25 & $9.43 *$ & 4.44 & 23.9 & 11.1 \\
\hline $\mathrm{Ca}(\mathrm{mg})$ & 1066 & 298 & $1097 *$ & 331 & 962 & 430.3 \\
\hline $\mathrm{Na}(\mathrm{mg})$ & 3068 & 567 & $3353^{*}$ & 677 & 2304 & 1080 \\
\hline Folate $(\mu \mathrm{g})$ & 306 & 115 & $341 *$ & 125 & 208 & 142 \\
\hline Vitamin C (mg) & 96.0 & 62.8 & $101 *$ & 67.5 & 77.1 & 104 \\
\hline $\mathrm{Fe}(\mathrm{mg})$ & 12.6 & 3.43 & $14.0 *$ & 3.80 & 9.17 & 4.86 \\
\hline Fibre (g) & 18.8 & 5.37 & $20.0 *$ & 5.37 & 16.2 & 8.74 \\
\hline
\end{tabular}

Mean values were significantly different from those for snacks: $* P<0.05$.

The mean number of all eating occasions was 4.3 per $\mathrm{d}$ with 2.6 the mean number of meals and 1.6 the mean number of snacks consumed per $\mathrm{d}$. There was no significant difference in the mean snack intake at weekend and weekdays. Meals and snacks contributed $74 \%$ and $25 \%$ respectively to total daily energy intake. Patterns of intakes from morning, afternoon and evening snacks were also explored. Morning, afternoon and evening snacks contributed 7\%,9\% and $12 \%$ respectively to total daily energy. No consistent trends were observed in nutrient intakes across all three snacking occasions; however, energy intakes for males were significantly greater than those for females $(P<0.05)$ at all snacks. Results therefore indicate that snacks make a considerable contribution to energy intake in Irish teens. However, snacks tended to make a less-favourable contribution to saturated fat and added sugar intakes than meals. Further research is needed to examine the types of foods consumed and whether these differences remained across tertiles of eating occasions.

1. Kerr MA, Rennie KL, McCaffrey TA et al. (2009) Br J Nutr 101, 122-131.

2. Stockman NK, Schenkel TC, Brown JN et al. (2004) Prev Med 41, 203-210.

3. Irish Universities Nutrition Alliance (2008) The National Teens Food Survey. http://www.iuna.net/index.php/research/teen-food-survey 